\title{
Probabilistic Cellular Automata
} for low temperature Ising model

\author{
Aldo Procacci ${ }^{1} \quad$ Benedetto Scoppola ${ }^{2} \quad$ Elisabetta Scoppola ${ }^{3}$ \\ July 11, 2018 \\ ${ }^{1}$ Departamento de Matemática Instituto de Ciências Exatas \\ Universidade Federal de Minas Gerais \\ Av. António Carlos, 6627 - Caixa Postal 702 30161-970 - Belo Horizonte - MG - BRASIL \\ aldo@mat .ufmg.br \\ 2 Dipartimento di Matematica, Università di Roma "Tor Vergata" \\ Via della Ricerca Scientifica - 00133 Roma, Italy \\ scoppola@mat.uniroma2. it \\ ${ }^{3}$ Dipartimento di Matematica e Fisica, Università Roma Tre \\ Largo San Murialdo, 1 - 00146 Roma, Italy \\ scoppola@mat.uniroma3.it
}

\begin{abstract}
We construct a parallel stochastic dynamics with invariant measure converging to the Gibbs measure of the low temperature Ising model. The proof of such convergence requires a polymer expansion based on suitably defined Peierls-type contours.
\end{abstract}




\section{Introduction}

Goal of the paper is to construct a parallel stochastic dynamics with invariant measure converging, in the thermodynamic limit, to the Gibbs measure of the Ising model at low temperature.

The parallel dynamics described in this paper is a homogeneous discrete time Markov chain on a product space $S^{\Lambda}=: \mathcal{X}$, where $S=\{-1,1\}$ and $\Lambda$ is a finite subset of $\mathbb{Z}^{d}$, with transition probabilities:

$$
P(\sigma, \tau)=\prod_{i \in \Lambda} P\left(\tau_{i} \mid \sigma\right) .
$$

This kind of dynamics are known in the literature as Probabilistic Cellular Automata (PCA).

Parallel dynamics is a challenging topic in Markov Chain Monte Carlo (MCMC) methods and statistical mechanics because they are very promising algorithms and the efficiency of parallel computing can be exploited in their simulation. From a theoretical point of view there are few results in the literature on the convergence to equilibrium of parallel dynamics. This is in general a difficult task, since the hight mobility related to parallelization, implies that it is much more complicated to individuate possible bottleneck or saddle configuration in the tunneling between different configurations on which the invariant measure can be concentrated than in the single spin flip dynamics. We mention here two example where the efficiency of parallel dynamics has been proved to be clearly higher than the efficiency of single spin flip dynamics. The first example is given in 5] where a control of the mixing time of an irreversible PCA related to the $2 \mathrm{~d}$ Ising model is given in a particular regime of low temperature in a finite box of side $L$ with periodic boundary conditions. In this case the mixing time turns out to be polynomial in $L$. A second example is the Swendsen-Wang dynamics on Ising model [22] (see also the review [19]). In this case the updating rule is not given in the form (11) of a PCA, but there is fast mixing because it is possible to update in a single step of the Markov chain a large amount of spins.

However there is a preliminary more difficult problem in the use of parallel dynamics in MCMC or in statistical mechanics: the control of their invariant measure. This is the reason why their use is not widespread, even if PCA have been introduced in Equilibrium Statistical Mechanics a long time ago, see for instance [17] and [10]. Invariant measures 
for infinite-volume PCA's may be non-Gibbsian ([8]) and even in the finite volume case it is usually completely different w.r.t. the invariant measure of Markov chain obtained by the random updating of a single site $i$, with the same probability $P\left(\tau_{i} \mid \sigma\right)$ used in (1).

In a previous paper [4], given a quite general spin system, a PCA was introduced with invariant measure converging, in the thermodynamic limit, to the Gibbs measure of the Ising model at high temperature. In the present paper we extend this result to the low temperature case.

We use here the same construction of the parallel dynamics introduced in [4] (see also [12], [16] and [9]). We recall here the main ingredients.

Given a spin configuration $\sigma \in \mathcal{X}$, start with a Hamiltonian of the form

$$
H(\sigma):=-\sum_{i, j} J_{i j} \sigma_{i} \sigma_{j}
$$

corresponding to the Gibbs measure

$$
\pi_{G}(\sigma)=\frac{e^{-H(\sigma)}}{\sum_{\sigma} e^{-H(\sigma)}}
$$

This Hamiltonian can be lifted to a Hamiltonian on $\mathcal{X} \times \mathcal{X}$, setting

$$
H(\sigma, \tau):=-\sum_{i, j} J_{i j} \sigma_{i} \tau_{j}+q \sum_{i}\left(1-\sigma_{i} \tau_{i}\right)
$$

with the property $H(\sigma, \sigma)=H(\sigma)$ and the PCA dynamics can be defined by

$$
P_{P C A}(\sigma, \tau)=\frac{e^{-H(\sigma, \tau)}}{\sum_{\tau} e^{-H(\sigma, \tau)}}
$$

In the symmetric case $J_{i j}=J_{j i}$ (and thus $H(\sigma, \tau)=H(\tau, \sigma)$ ) it is immediate to prove that this is a reversible PCA with invariant measure

$$
\pi_{P C A}(\sigma)=\frac{\sum_{\tau} e^{-H(\sigma, \tau)}}{\sum_{\tau, \tau^{\prime}} e^{-H\left(\tau, \tau^{\prime}\right)}}
$$

We note that pair hamiltonians were already present in the literature but their use was essentially related to generalizations of the detail balance condition. See for instance in [21] the notion of "approximately reversible non degenerate" Markov chain. In these 
cases a pair hamiltonian was introduced in order to write

$$
P(\sigma, \tau) \propto e^{-[H(\sigma, \tau)-H(\sigma)]}
$$

so that reversibility w.r.t. the Gibbs measure was immediately related to the symmetry condition $H(\sigma, \tau)=H(\tau, \sigma)$.

Here the pair hamiltonian $H(\sigma, \tau)$ has a different role since it is the necessary ingredient to define the dynamics. More precisely, to define the dynamics we want to consider a Gibbs measure $\mu(\sigma, \tau) \propto e^{-H(\sigma, \tau)}$ on the space of pairs of configurations $(\sigma, \tau) \in \mathcal{X}^{2}$ instead of the Gibbs measure for single configurations, since pairs of configurations are possible moves of the dynamics. The "lifting" given by the definition of pair Hamiltonian (2) is due to the quadratic form of $H(\sigma)$ so that it is possible to consider $H(\sigma)=H(\sigma, \sigma)$.

Once the pair hamiltonian is given, it is natural to define the measure on $\mathcal{X} \times \mathcal{X}$ :

$$
\mu(\sigma, \tau)=\frac{e^{-H(\sigma, \tau)}}{Z} \quad \text { with } \quad Z=\sum_{\sigma, \tau} e^{-H(\sigma, \tau)} .
$$

The marginal of $\mu(\sigma, \tau)$ is the measure on $\mathcal{X}$ :

$$
\sum_{\tau} \frac{e^{-H(\sigma, \tau)}}{Z}=: \nu(\sigma) \equiv \frac{Z_{\sigma}}{Z}
$$

and if $H(\sigma, \tau)=H(\tau, \sigma)$, i.e., in the reversible case, we have $\mu(\sigma, \tau)=\mu(\tau, \sigma)=\mu(\{\sigma, \tau\})$ and $\nu(\sigma)=\sum_{\tau} \frac{e^{-H(\tau, \sigma)}}{Z}$.

For a given $\sigma \in \mathcal{X}$ the transition probability of the chain associated to the measure $\mu$ is given by

$$
P(\sigma, \tau)=P((\sigma, \tau) \mid \sigma)=\frac{\mu(\sigma, \tau)}{\nu(\sigma)}=\frac{e^{-H(\sigma, \tau)}}{Z_{\sigma}} \equiv P_{P C A}(\sigma, \tau)
$$

and $\nu(\sigma)=\frac{Z_{\sigma}}{Z}=\pi_{P C A}(\sigma)$ is clearly its invariant measure.

The irreversible case, when $H(\sigma, \tau) \neq H(\tau, \sigma)$, is less trivial. By considering periodic boundary condition, in the completely asymmetric case, see (20), is possible to prove that

$$
\nu(\sigma):=\sum_{\tau} \frac{e^{-H(\sigma, \tau)}}{Z}=\sum_{\tau} \frac{e^{-H(\tau, \sigma)}}{Z}
$$

so that again $\nu$ is the marginal measure and equation (5) is again the natural definition of the dynamics. 
Note that the same construction holds here in the reversible and non-reversible case, at least in the case of periodic boundary conditions. This is an interesting feature of our approach, introducing a unique promising language to treat equilibrium and nonequilibrium statistical mechanics.

Define the total variation distance, or $L_{1}$ distance, between $\pi_{G}$ and $\pi_{P C A}$ as

$$
\left\|\pi_{P C A}-\pi_{G}\right\|_{T V}=\frac{1}{2} \sum_{\sigma \in \mathcal{X}}\left|\pi_{P C A}(\sigma)-\pi_{G}(\sigma)\right|
$$

The parameter $q$ controls the average number of spin-flips in a single step of the dynamics. It was proved in [4] that, defining $\delta:=e^{-2 q}$, if $\delta=\delta(|\Lambda|)$ is such that $\lim _{|\Lambda| \rightarrow \infty} \delta^{2}|\Lambda|=0$ and if the temperature is sufficiently high (i.e. $J_{i, j}$ sufficiently small) then

$$
\lim _{|\Lambda| \rightarrow \infty}\left\|\pi_{P C A}-\pi_{G}\right\|_{T V}=0 .
$$

In the present paper we use completely different tools to prove a similar result in the low temperature regime. Indeed we will use a polymer expansion based on suitably defined Peierls-type contours. The cluster expansion techniques involved in the present paper are quite robust. We present the results in the simple case of two dimensions:

- with plus boundary conditions in the reversible case;

- with periodic boundary conditions in the reversible case;

- with periodic boundary conditions in the irreversible case.

As far as the choice of parameters is concerned, we are in a quite general regime. We need again the hypothesis $\lim _{|\Lambda| \rightarrow \infty} \delta^{2}|\Lambda|=0$ but the low temperature regime is not related to a particular asymptotic in the limit $|\Lambda| \rightarrow \infty$. In the irreversible case a similar result was obtained in [5] but in a very particular regime of low temperature, where inverse temperature suitably increases with the volume.

We finally remark that in this paper estimates are not optimized.

\subsection{Definitions}

Henceforth $\Lambda$ denotes a two-dimensional $L \times L$ square lattice in $\mathbb{Z}^{2}$ and $B_{\Lambda}$ denotes the set of all nearest neighbors in $\Lambda$, i.e. $B_{\Lambda}=\{\langle i, j\rangle: i, j \in \Lambda,|i-j|=1\}$ with $|i-j|$ 
being the usual lattice distance in $\mathbb{Z}^{d}$. We denote by $\partial^{\text {ext }} \Lambda\left(\partial^{i n t} \Lambda\right)$ the external (internal) boundary of $\Lambda$, i.e. $\partial^{e x t} \Lambda=\left\{i \in \mathbb{Z}^{2} \backslash \Lambda: \exists j \in \Lambda\right.$ s.t. $\left.|i-j|=1\right\}\left(\partial^{i n t} \Lambda=\{i \in \Lambda: \exists j \in\right.$ $\mathbb{Z}^{2} \backslash \Lambda$ s.t. $\left.\left.|i-j|=1\right\}\right)$ and we set $\bar{\Lambda}=\Lambda \cup \partial^{\text {ext }} \Lambda$

We set $B_{\Lambda}^{p e r}=\{\langle i, j\rangle: i, j \in \Lambda$ and either $|i-j|=1$ or $|i-j|=L-1\}$. Namely, $B_{\Lambda}^{\text {per }}$ is the set of all nearest neighbors in $\Lambda$ plus the pairs of sites at opposite faces of the square $\Lambda$, so that the pair $\left(\Lambda, B_{\Lambda}^{p e r}\right)$ is homeomorphic to the two-dimensional discrete torus $\left(\mathbb{Z} /(L \mathbb{Z})^{2}\right.$.

We set $B_{\Lambda}^{+}=\{\langle i, j\rangle: i, j \in \bar{\Lambda}:|i-j|=1\}$, i.e. $B_{\Lambda}^{+}$is the set of all nearest neighbors in $\bar{\Lambda}$. We finally recall that $\mathcal{X}$ denotes the set of spin configurations in $\Lambda$., i.e., $\mathcal{X}$ is the set of all functions $\sigma: \Lambda \rightarrow\{-1,1\}$.

In both cases of periodic and + b.c. define the Ising hamiltonian with interaction $J>0$ as follows. Given $\sigma \in \mathcal{X}$,

$$
H^{p e r}(\sigma)=-J \sum_{\langle i, j\rangle \in B_{\Lambda}^{p e r}} \sigma_{i} \sigma_{j}, \quad H^{+}(\sigma)=-J \sum_{\langle i, j\rangle \in B_{\Lambda}^{+}} \sigma_{i} \sigma_{j}
$$

where by convention $\sigma_{i}=+1$ when $i \in \partial^{e x t} \Lambda$.

Using the notation $H^{*}(\sigma)$ with either $*=$ per or $*=+$, we denote

$$
w_{G}^{*}(\sigma)=e^{-H^{*}(\sigma)}, \quad Z_{G}^{*}=\sum_{\sigma \in \mathcal{X}} e^{-H^{*}(\sigma)}
$$

so that the standard Gibbs measure with $*=$ per,+ is

$$
\pi_{G}^{*}(\sigma)=\frac{w_{G}^{*}(\sigma)}{Z_{G}^{*}}
$$

We now define, for every pair of configurations $\sigma, \tau \in \mathcal{X}$ the following pair Hamiltonians for periodic and + b.c. respectively:

$$
\begin{aligned}
& H^{p e r}(\sigma, \tau)=-\frac{J}{2} \sum_{i \in \Lambda} \sum_{\substack{j \in \Lambda \\
\langle i, j\rangle \in B_{\Lambda}^{p e r}}} \sigma_{i} \tau_{j}+q \sum_{i \in \Lambda}\left(1-\sigma_{i} \tau_{i}\right) \\
& H^{+}(\sigma, \tau)=-\frac{J}{2} \sum_{i \in \Lambda} \sum_{\substack{j \in \Lambda: \\
\langle i, j\rangle \in B_{\Lambda}}} \sigma_{i} \tau_{j}-\frac{J}{2} \sum_{i \in \partial^{i n t}} \sum_{\substack{j_{j} \partial^{e x t} t_{\Lambda} \\
|i-j|=1}}\left(\sigma_{i}+\tau_{i}\right)+q \sum_{i \in \Lambda}\left(1-\sigma_{i} \tau_{i}\right)
\end{aligned}
$$

where $q>0$ is a volume-dependent parameter that we will choose later (see Theorem 1.1 
ahead). Note that $\sum_{\substack{j \in \partial^{e x t} \Lambda: \\|i-j|=1}}$ is equal to one in each site in $\partial^{i n t} \Lambda$ which is not a corner and is equal to 2 in the four corners of $\partial^{\text {int }} \Lambda$.

Clearly, for $*=+$, per, $H^{*}(\sigma, \tau)$ is symmetric, i.e. $H^{*}(\sigma, \tau)=H^{*}(\tau, \sigma)$. Moreover observing that $H^{\text {per }}(\sigma)$ and $H^{+}(\sigma)$ can be rewritten respectively as

$$
\begin{aligned}
& H^{p e r}(\sigma)=-\frac{J}{2} \sum_{i \in \Lambda} \sum_{\substack{j \in \Lambda \\
\langle i, j\rangle \in B_{\Lambda}^{p e r}}} \sigma_{i} \sigma_{j} \\
& H^{+}(\sigma)=-\frac{J}{2} \sum_{i \in \Lambda} \sum_{\substack{j \in \Lambda \\
\langle i, j\rangle \in B_{\Lambda}}} \sigma_{i} \sigma_{j}-J \sum_{i \in \partial^{i n t}} \sum_{\substack{j \in \partial^{e x t} \Lambda \\
|i-j|=1}} \sigma_{i}
\end{aligned}
$$

we also get immediately that $H^{*}(\sigma, \sigma)=H^{*}(\sigma)$.

Let us write

$$
\partial_{i}=\frac{J}{2} \mathbf{1}_{\left\{i \in \partial^{i n t} \Lambda\right\}} \sum_{\substack{j \in \partial^{e x t} \Lambda: \\|i-j|=1}} 1, \quad G(\sigma)=\sum_{i \in \Lambda} \partial_{i} \sigma_{i}
$$

and define

$$
h_{i}^{+}(\sigma)=\frac{J}{2} \sum_{\substack{j \in \Lambda_{0} \\\langle i, j\rangle \in B_{\Lambda}}} \sigma_{j}+\partial_{i}, \quad h_{i}^{p e r}(\sigma)=\frac{J}{2} \sum_{\substack{j \in \Lambda_{i} \\\langle i, j\rangle \in B_{\Lambda}^{p e r}}} \sigma_{j} .
$$

Then we can rewrite

$$
H^{*}(\sigma, \tau)=-\sum_{i \in \Lambda}\left(h_{i}^{*}(\sigma)+\sigma_{i} q\right) \tau_{i}-G^{*}(\sigma)+q|\Lambda|
$$

with $G^{+}(\sigma)=G(\sigma)$ and $G^{p e r}(\sigma)=0$. We can now define parallel dynamics, that we will call $P C A$ dynamics, with the following transition probabilities, for $*=p e r,+$

$$
P_{P C A}^{*}(\sigma, \tau)=\frac{e^{-H^{*}(\sigma, \tau)}}{\sum_{\tau \in \mathcal{X}} e^{-H^{*}(\sigma, \tau)}}
$$

It is a standard task to show that this PCA dynamics is reversible with respect to the measure

$$
\pi_{P C A}^{*}(\sigma)=\frac{\sum_{\tau \in \mathcal{X}} e^{-H^{*}(\sigma, \tau)}}{\sum_{\tau, \tau^{\prime} \in \mathcal{X}} e^{-H^{*}\left(\tau, \tau^{\prime}\right)}}
$$

Due to (17), the transition probabilities of this Markov chain can be written as a product of the transition probabilities of each component $\tau_{i}$ of the new configuration $\tau$, as usual 
for PCAs:

$$
P_{P C A}^{*}(\sigma, \tau)=\prod_{i \in \Lambda} P^{*}\left(\tau_{i} \mid \sigma\right)
$$

with

$$
P^{*}\left(\tau_{i} \mid \sigma\right)=\frac{e^{\left(h_{i}^{*}(\sigma)+\sigma_{i} q\right) \tau_{i}}}{2 \cosh \left(h_{i}^{*}(\sigma)+\sigma_{i} q\right)} .
$$

In the case of periodic boundary condition we will also consider an irreversible parallel dynamics, denoted with $I$ for irreversible, by considering the following pair hamiltonian

$$
H^{I}(\sigma, \tau)=-\sum_{i \in \Lambda}\left[J \sigma_{i}\left(\tau_{i \uparrow}+\tau_{i} \rightarrow\right)+q \sigma_{i} \tau_{i}\right]=-\sum_{i \in \Lambda}\left[J \tau_{i}\left(\sigma_{i \downarrow}+\sigma_{i^{\leftarrow}}\right)+q \sigma_{i} \tau_{i}\right]
$$

where $i^{\uparrow}, i^{\rightarrow}, i^{\downarrow}, i^{\leftarrow}$ are respectively the up, right, down, left neighbors of the site $i$ on the torus $\left(\Lambda, B_{\Lambda}^{\text {per }}\right)$. We have $H^{I}(\sigma, \sigma)=H^{\text {per }}(\sigma)-q|\Lambda|$ but note that now $H^{I}(\sigma, \tau) \neq$ $H^{I}(\tau, \sigma)$. However, as shown in [5, 16, the following weak symmetry condition (dynamical balance) holds

$$
\sum_{\tau \in \mathcal{X}} e^{-H^{I}(\sigma, \tau)}=\sum_{\tau \in \mathcal{X}} e^{-H^{I}(\tau, \sigma)}
$$

so that the parallel dynamics defined by

$$
P_{P C A}^{I}(\sigma, \tau)=\prod_{i \in \Lambda} \frac{\exp \left\{\tau_{i}\left[J\left(\sigma_{i \downarrow}+\sigma_{i \leftarrow}\right)+q \sigma_{i}\right]\right\}}{2 \cosh \left(J\left(\sigma_{i \downarrow}+\sigma_{i^{\leftarrow}}\right)+q \sigma_{i}\right)}
$$

is irreversible with a unique stationary distribution $\pi_{P C A}^{I}$ given by

$$
\pi_{P C A}^{I}(\sigma):=\frac{Z_{\sigma}^{I}}{Z_{P C A}^{I}}, \quad Z_{\sigma}^{I}=\sum_{\tau \in \mathcal{X}} e^{-H^{I}(\sigma, \tau)}
$$

with $Z_{P C A}^{I}:=\sum_{\sigma} Z_{\sigma}^{I}$.

\subsection{Results}

By using the previous definitions we can now state our general results. The first theorem states that the stationary measure of the reversible PCA and the Gibbs measure are equivalent in the thermodynamical limit.

Theorem 1.1 Set $\delta=e^{-2 q}$, and let $\delta$ be such that

$$
\lim _{|\Lambda| \rightarrow \infty} \delta^{2}|\Lambda|=0
$$


then, there exist $J_{c}$ such that for any $J>J_{c}$

$$
\lim _{|\Lambda| \rightarrow \infty}\left\|\pi_{P C A}^{*}-\pi_{G}^{*}\right\|_{T V}=0 \quad *=+, p e r
$$

The second theorem deals with the irreversible case, with periodic boundary conditions

Theorem 1.2 Under the assumption (22), there exist $J_{c}$ such that for any $J>J_{c}$

$$
\lim _{|\Lambda| \rightarrow \infty}\left\|\pi_{P C A}^{I}-\pi_{G}^{p e r}\right\|_{T V}=0
$$

Remark 1.3 Let us make some comments on the results and its relations with the similar results obtained in [4] and [5].

i) First note that (22) corresponds to say that the parameter $q$ goes to infinity faster than $\frac{1}{4} \ln |\Lambda|$. This hypothesis was also assumed in [4].

ii) As far as Theorem 1.1 is concerned, as mentioned in the introduction, a similar result was obtained in [4] but in the opposite regime of high temperature (uniqueness of phase for the Gibbs measure).

iii) As far as Theorem 1.2 is concerned a similar result was obtained in [5] but with a very particular choice of the parameters. In that paper indeed a low temperature regime was defined by fixing $J(L)$ and $q(L)$ as function of the side $L$ of the square $\Lambda$. In the present paper we want to stress that we are in a true low temperature regime, i.e., we prove the convergence of the invariant measures of the PCA to the Gibbs measure in the thermodynamical limit for any $J$ large enough provided that the hypothesis (22) on the parameter $q$ is satisfied.

iv) Actually the idea of the proof of Theorems 1.1 and 1.2 follows the same line of the similar results in [4] and [5]. The strategy is to prove that the following inequality holds

$$
\left\|\pi_{P C A}^{*}-\pi_{G}^{*}\right\|_{T V}=\mathcal{O}\left(\delta|\Lambda|^{1 / 2}\right)
$$

and then immediately concluding the proof by using the hypothesis (22). However the proof of (25), is based on Lemmata 2.1] and 2.2 which are completely different w.r.t. the tools used in the previous papers [4], [5]. 


\section{Proof of Theorems 1.1 and 1.2}

Let us start with Theorem 1.1. We first prove that for any fixed $J>J_{c}$ there exist $\delta_{J}$ such that for any $\delta<\delta_{J}(25)$ holds.

Let, for $*=p e r,+$

$$
w_{P C A}^{*}(\sigma)=\sum_{\tau \in \mathcal{X}} e^{-H^{*}(\sigma, \tau)}
$$

Then, recalling (17), we have (modulo the constant $e^{-q|\Lambda|}$ which cancels out in the ratio $\left.w_{P C A}^{*}(\sigma) / \sum_{\sigma} w_{P C A}^{*}(\sigma)\right)$

$$
w_{P C A}^{*}(\sigma)=e^{G^{*}(\sigma)} \sum_{\tau \in \mathcal{X}} e^{\left.\sum_{i \in \Lambda}\left[\left(h_{i}^{*}(\sigma)+q \sigma_{i}\right) \tau_{i}\right)\right]}
$$

We now rewrite the sum on $\tau \in \mathcal{X}$ in the following way. Given $\sigma \in \mathcal{X}$, to sum over all $\tau \in \mathcal{X}$ is the same as to sum over all subset $I \subset \Lambda$ such that $\tau_{i}=-\sigma_{i}$ if $i \in I$ while $\tau_{i}=\sigma_{i}$ otherwise. Hence we can write

$$
\begin{gathered}
w_{P C A}^{*}(\sigma)=e^{G^{*}(\sigma)} \sum_{I \subset \Lambda} e^{\sum_{i \in \Lambda} h_{i}^{*}(\sigma) \sigma_{i}-2 \sum_{i \in I} h_{i}^{*}(\sigma) \sigma_{i}-2 q|I|}= \\
=e^{\sum_{i \in \Lambda} h_{i}^{*}(\sigma) \sigma_{i}+G^{*}(\sigma)} \sum_{I \subset \Lambda} \prod_{i \in I} e^{-2 h_{i}^{*}(\sigma) \sigma_{i}-2 q}=e^{\sum_{i \in \Lambda} h_{i}^{*}(\sigma) \sigma_{i}+G^{*}(\sigma)} \prod_{i \in \Lambda}\left(1+\delta \phi_{i}^{*}\right)
\end{gathered}
$$

where, recalling that $\delta=e^{-2 q}$, we have defined

$$
\phi_{i}^{*}=e^{-2\left(h_{i}^{*}(\sigma)\right) \sigma_{i}}
$$

By definitions (13), (14), (15) and (16) it is easy to check that

$$
\sum_{i \in \Lambda} h_{i}^{*}(\sigma) \sigma_{i}+G^{*}(\sigma)=-H^{*}(\sigma)
$$

Therefore, recalling (9) and setting

$$
f^{*}(\sigma)=\prod_{i \in \Lambda}\left(1+\delta \phi_{i}^{*}\right),
$$

we get

$$
w_{P C A}^{*}(\sigma)=w_{G}^{*}(\sigma) f^{*}(\sigma)
$$


and therefore, by definitions (26), (10) and (19) we have

$$
\pi_{P C A}^{*}(\sigma)=\frac{w_{P C A}^{*}(\sigma)}{\sum_{\sigma \in \mathcal{X}} w_{P C A}^{*}(\sigma)}=\frac{w_{G}^{*}(\sigma) f^{*}(\sigma)}{\sum_{\sigma \in \mathcal{X}} w_{G}^{*}(\sigma) f^{*}(\sigma)}=\frac{\frac{w_{G}^{*}(\sigma)}{Z_{G}^{*}} f^{*}(\sigma)}{\sum_{\sigma \in \mathcal{X}} \frac{w_{G}^{*}(\sigma)}{Z_{G}^{*}} f^{*}(\sigma)}=\frac{\pi_{G}^{*}(\sigma) f^{*}(\sigma)}{\pi_{G}^{*}\left(f^{*}\right)}
$$

Hence

$\left\|\pi_{P C A}^{*}-\pi_{G}^{*}\right\|_{T V}=\sum_{\sigma} \pi_{G}^{*}(\sigma)\left|\frac{f^{*}(\sigma)}{\pi_{G}^{*}\left(f^{*}\right)}-1\right|=\pi_{G}^{*}\left(\left|\frac{f^{*}(\sigma)}{\pi_{G}^{*}\left(f^{*}\right)}-1\right|\right) \leq \frac{\left(\operatorname{var}_{\pi_{G}^{*}}\left(f^{*}\right)\right)^{1 / 2}}{\pi_{G}^{*}\left(f^{*}\right)}=\left(\Delta^{*}(\delta)\right)^{1 / 2}$

with

$$
\Delta^{*}(\delta)=\frac{\pi_{G}\left(\left(f^{*}\right)^{2}\right)}{\left(\pi_{G}\left(f^{*}\right)\right)^{2}}-1
$$

By writing

$$
\Delta^{*}(\delta)=\exp \left[\ln \pi_{G}^{*}\left(\left(f^{*}\right)^{2}\right)-2 \ln \pi^{G}\left(f^{*}\right)\right]-1
$$

Theorem 1.1 is proved by the following:

Lemma 2.1 There exists $J_{c}$ such that for any $J>J_{c}$

$$
\begin{aligned}
& \text { i) } \frac{\ln \pi_{G}^{*}\left(\left(f^{*}\right)^{2}\right)}{|\Lambda|} \text { and } \frac{\ln \pi_{G}^{*}\left(f^{*}\right)}{|\Lambda|} \text { are analytical functions of } \delta \text { for }|\delta|<\delta_{J} . \\
& \text { ii) } \frac{\ln \pi_{G}^{*}\left(\left(f^{*}\right)^{2}\right)}{|\Lambda|}-2 \frac{\ln \pi_{G}^{*}\left(f^{*}\right)}{|\Lambda|}=O\left(\delta^{2}\right)
\end{aligned}
$$

\section{Proof}

Part i)

We will show the analyticity of $\frac{\ln \pi_{G}^{*}\left(\left(f^{*}\right)^{2}\right)}{|\Lambda|}$ and $\frac{\ln \pi_{G}^{*}\left(f^{*}\right)}{|\Lambda|}$ by showing that both these quantities may be written as partition functions of an abstract polymer gas. Then the analiticity will follow by standard cluster expansion, see [13, 1, 17.

We have

$$
\pi_{G}^{*}\left(\left(f^{*}\right)^{k}\right)=\frac{1}{Z_{G}^{+}} \sum_{\sigma \in \mathcal{X}} e^{-H^{*}(\sigma)} \prod_{i \in \Lambda}\left(1+\delta \phi_{i}^{*}\right)^{k}, \quad k=1,2
$$

and, recalling (16), (28), note that

$$
\phi_{i}^{*}=\exp \left\{-2 h_{i}^{*}(\sigma) \sigma_{i}\right\}=\exp \left\{-J \sum_{\substack{j \in \Lambda^{*} \\\langle i, j\rangle \in B_{\Lambda}^{*}}} \sigma_{i} \sigma_{j}\right\}
$$

with $\Lambda^{+}=\bar{\Lambda}, \Lambda^{\text {per }}=\Lambda$ and with the usual convention that $\sigma_{j}=+1$ when $j \in \partial_{\Lambda}^{e x t}$. 
We first consider $\pi_{G}^{+}\left(\left(f^{+}\right)^{k}\right), k=1,2$. Then, by (34), we can write

$$
\pi_{G}^{+}\left(\left(f^{+}\right)^{k}\right)=\frac{1}{Z_{G}^{+}} \sum_{\sigma \in \mathcal{X}} \exp \left\{J \sum_{\langle i, j\rangle \in B_{\Lambda}^{+}} \sigma_{i} \sigma_{j}\right\} \prod_{i \in \Lambda}\left(1+\delta \exp \left\{-J \sum_{j \in \bar{\Lambda},|j-i|=1} \sigma_{i} \sigma_{j}\right\}\right)^{k}
$$

with the convention that $\sigma_{j}=+1$ whenever $j \in \partial^{e x t} \Lambda$.

We rewrite the 1.h.s. of (35) via a standard Peierls contour gas. Following the usual construction, for a fixed configuration $\sigma$, let $\Gamma$ be the set of unit segments perpendicular to the center of each bond of nearest neighbors in $\bar{\Lambda}$ having opposite spins at its extremes (again with the convention that $\sigma_{i}=1$ if $i \in \partial^{e x t} \Lambda$ ). Any unit segment $e \in \Gamma$ is a nearest neighbor bond of a $(L+1) \times(L+1)$ square with vertices in the dual unit square lattice $\mathbb{Z}^{* 2}$ (translated by the vector $\left(\frac{1}{2}, \frac{1}{2}\right)$ respect to the original lattice $\mathbb{Z}^{2}$ ). As far as + b.c are concerned, the correspondence $\sigma \mapsto \Gamma$ is one-to-one and the unit segments of $\Gamma$ form a collection of closed polygons which separate regions where the spins are positive from regions where they are negative. These polygons possibly intercept themselves in such a way that the degree of each vertex is even. Let us denote by $\mathcal{G}_{\Lambda}$ the set of all possible $\Gamma$.

$$
J \sum_{\langle i, j\rangle \in B_{\bar{\Lambda}}} \sigma_{i} \sigma_{j}=\left|B_{\bar{\Lambda}}\right|-2 J|\Gamma|
$$

Moreover, calling $l_{s}(\Gamma)$, for $s=1, \ldots, 4$, the set of vertices $i \in \Lambda$ having exactly $s$ edges $\langle i, j\rangle$ with dual in $\Gamma$, we have

$$
\begin{gathered}
\prod_{i \in \Lambda}\left(1+\delta \exp \left\{-J \sum_{j \in \bar{\Lambda},|j-i|=1} \sigma_{i} \sigma_{j}\right\}\right)^{k}=\prod_{i \in \Lambda \backslash \cup_{s=1}^{4} l_{s}(\Gamma)}\left(1+\delta e^{-4 J}\right)^{k} \times \prod_{i \in l_{1}(\Gamma)}\left(1+\delta e^{-2 J}\right)^{k} \times \\
\times \prod_{i \in l_{2}(\Gamma)}(1+\delta)^{k} \times \prod_{i \in l_{3}(\Gamma)}\left(1+\delta e^{+2 J}\right)^{k} \times \prod_{i \in l_{4}(\Gamma)}\left(1+\delta e^{+4 J}\right)^{k}
\end{gathered}
$$

Therefore (35) can be rewritten as follows

$$
\pi_{G}^{+}\left(\left(f^{+}\right)^{k}\right)=\frac{1}{Z_{G}^{+}} e^{J\left|B_{\bar{\Lambda}}\right|}\left(1+\delta e^{-4 J}\right)^{k|\Lambda|} \Xi_{\Lambda}^{+(k)}(J, \delta)
$$

where

$$
\Xi_{\Lambda}^{+(k)}(J, \delta)=\sum_{\Gamma \in \mathcal{G}_{\Lambda}}\left[e^{-2 J|\Gamma|} \xi_{k}(\Gamma)\right]
$$


with

$$
\xi_{k}(\Gamma)=\left[\frac{1+\delta e^{-2 J}}{1+\delta e^{-4 J}}\right]^{k\left|l_{1}(\Gamma)\right|}\left[\frac{1+\delta}{1+\delta e^{-4 J}}\right]^{k\left|l_{2}(\Gamma)\right|}\left[\frac{1+\delta e^{+2 J}}{1+\delta e^{-4 J}}\right]^{k\left|l_{3}(\Gamma)\right|}\left[\frac{1+\delta e^{+4 J}}{1+\delta e^{-4 J}}\right]^{k\left|l_{4}(\Gamma)\right|}
$$

From (36) we obtain that

$$
\frac{1}{|\Lambda|} \ln \pi_{G}^{+}\left(\left(f^{+}\right)^{k}\right)=-\frac{\ln Z_{G}^{+}}{|\Lambda|}+J \frac{\left|B_{\bar{\Lambda}}\right|}{|\Lambda|}+k \ln \left(1+\delta e^{-4 J}\right)+\frac{1}{|\Lambda|} \ln \Xi_{\Lambda}^{+(k)}(J, \delta)
$$

The first two terms of the 1.h.s. of identity above do not depend on $\delta$ and are uniformly bounded as $\Lambda \rightarrow \infty$ and the third term is analytic in $\delta$ and independent of $\Lambda$. It thus remains to be analyzed the term $\frac{1}{|\Lambda|} \ln \Xi_{\Lambda}^{+(k)}(J, \delta)$. In order to do that, we regard each $\Gamma \in \mathcal{G}_{\Lambda}$ as the disjount union of its suitably defined $p$-connected components $\gamma_{1}, \ldots, \gamma_{n}$ in such a way that any vertex of $l_{s}(\Gamma), s=1, \ldots, 4$, is associated to at most one p-connected component $\gamma_{i}$. We introduce the notion of p-connection as an extension of the usual notion of connection. Namely we define two unit segments of $\Gamma$ as $p$-connected if they share a common vertex or if they are parallel and at distance 1 . We denote by $\mathcal{P}_{\Lambda}$ the set of all such p-connected components, i.e., $\gamma_{i} \in \mathcal{P}_{\Lambda}$. For $\gamma, \gamma^{\prime} \in \mathcal{P}_{\Lambda}$ we write $\gamma \sim \gamma^{\prime}$ if $\gamma \cup \gamma^{\prime} \notin \mathcal{P}_{\Lambda}$. With these notations (37) can be written as follows

$$
\Xi_{\Lambda}^{+(k)}(J, \delta)=\sum_{n \geq 0} \frac{1}{n !} \sum_{\substack{\left(\gamma_{1}, \ldots, \gamma_{n}\right) \in \mathcal{P}_{\Lambda}^{n} \\ \gamma_{i} \sim \gamma_{j}}} \prod_{i=1}^{n}\left[e^{-2 J\left|\gamma_{i}\right|} \xi_{k}\left(\gamma_{i}\right)\right]
$$

where the $n=0$ contribution is 1 , and it corresponds of course to the configuration $\sigma=1$.

The r.h.s. of (39) is the grand canonical partition function of a hard-core polymer gas, where the polymers are the elements of $\mathcal{P}_{\Lambda}$ defined above and their activity $\rho_{k}(\gamma)$ is

$$
\rho_{k}(\gamma)=\xi_{k}(\gamma) e^{-2 J|\gamma|}
$$

It is well known that the logarithm of $\Xi_{\Lambda}^{+(k)}(J, \delta)$ divided by $|\Lambda|$ can be written as an absolutely convergent series uniformly in $\Lambda$ for $\rho_{k}(\gamma)$ sufficiently small. For the sake of simplicity, and since we are not seeking optimal bounds, we will use the Kotecky-Preiss (KP) condition [13] (see also [1], [7] for better conditions). In its general form, the KP condition reads

$$
\sum_{\gamma \nsim \gamma^{\prime}} \rho(\gamma) e^{a(\gamma)} \leq a\left(\gamma^{\prime}\right)
$$


where $a(\gamma)$ is any positive function of $\gamma$. Choosing $a(\gamma)=a|\gamma|, a>0$, and noting that

$$
\sum_{\gamma \nsim \gamma^{\prime}} \rho(\gamma) e^{a|\gamma|} \leq 3\left|\gamma^{\prime}\right| \sup _{x \in \mathbb{Z}^{2}} \sum_{\gamma \ni x} \rho(\gamma) e^{a|\gamma|}
$$

we have that equation (41) is satisfied if

$$
\sup _{x \in \Lambda} \sum_{\substack{\gamma \in \mathcal{P} \\ x \in \gamma}} \rho_{k}(\gamma) e^{a|\gamma|} \leq \frac{a}{3}
$$

where $\mathcal{P}$ is the set of all p-connected contours in $\mathbb{Z}^{2}$. The factor 3 in the r.h.s. of (42) comes from the fact that the contour $\gamma$ is anchored to $\gamma^{\prime}$ if there exists a vertex $x$ of $\gamma^{\prime}$ of degree 2 in $\gamma^{\prime}$ such that either $x$ is also a vertex of $\gamma$ or the edge exiting from $x$ in $\gamma^{\prime}$, when $\gamma^{\prime}$ is traveled in a given direction, is parallel and at distance 1 to an edge of $\gamma$. In the latter case there are two possible choices, hence the factor 3 .

As we said in the introduction it is not our intention to look for optimal estimate. We thus choose $a=1$ (which is not optimal) and hence condition (43) becomes

$$
\sup _{x \in \mathbb{Z}^{2}} \sum_{\substack{\gamma \in \mathcal{P} \\ x \in \gamma}} \rho_{k}(\gamma) e^{|\gamma|} \leq \frac{1}{3}
$$

To check (44), let first obtain an upper bound for $\rho_{k}(\gamma)$. Recalling the definition (38), we get, for $|\delta|<e^{4 J}$

$$
\rho_{k}(\gamma) \leq e^{-2 J|\gamma|}\left[\frac{1+|\delta| e^{+4 J}}{1-|\delta| e^{-4 J}}\right]^{k \sum_{s=1}^{4}\left|l_{s}(\gamma)\right|}
$$

Observing that

$$
\sum_{s=1}^{4}\left|l_{s}(\gamma)\right| \leq 2|\gamma|
$$

we get, for any $k=1,2$

$$
\rho_{k}(\gamma) \leq e^{-2 J|\gamma|}\left[\frac{1+|\delta| e^{+4 J}}{1-|\delta| e^{-4 J}}\right]^{2 k|\gamma|} \leq\left(e^{-2 J}\left[\frac{1+|\delta| e^{+4 J}}{1-|\delta| e^{-4 J}}\right]^{4}\right)^{|\gamma|}
$$

Let us call

$$
A(J, \delta)=e^{-2 J}\left[\frac{1+|\delta| e^{+4 J}}{1-|\delta| e^{-4 J}}\right]^{4}
$$

When the p-connected contour $\gamma \in \mathcal{P}_{\Lambda}$ is composed by several different connected components we define a new connected contour by adding edges between adjacent contours. 
We define a rule. For instance we order the vertices of $\Lambda$ and we consider the consequent order of edges with lexicographic ordering. Given a contour $\gamma$, we add to the contour pairs of edges corresponding to the set of smallest edges in order to obtain a connected graph. The resulting graph $\bar{\gamma}$ is then a connected graph with possible multiple edges (the added pairs), with even degree at each vertex, with maximal degree 4. Since each connected component of $\gamma$ has at least 4 edges, the number of added pairs of edges is at most $\frac{|\gamma|}{4}$.

Then

$$
\sup _{x \in \mathbb{Z}^{2}} \sum_{\gamma \ni x} \rho_{k}(\gamma) e^{|\gamma|} \leq \sum_{n \geq 4} A(J, \delta)^{n} e^{n} \sum_{\substack{\hat{\gamma} \ni 0 \\|\gamma|=n}} 1 \leq \sum_{n \geq 4}\left[e \cdot 3^{3 / 2} A(J, \delta)\right]^{n}
$$

Here the factor $3^{3 n / 2}$ comes from the estimate of the number of graphs $\bar{\gamma}$ of $3 n / 2$ edges passing through a fixed vertex. Indeed the graph $\bar{\gamma}$ has a Eulerian circuit and, as usual, each new step has three possible choices. Therefore (44) is surely fulfilled for $k=1,2$ if

$$
\frac{\left[e \cdot 3^{3 / 2} A(J, \delta)\right]^{4}}{1-e \cdot 3^{3 / 2} A(J, \delta)} \leq \frac{1}{3} \quad \Longrightarrow \quad A(J, \delta)<\frac{1}{2 e 3^{3 / 2}}
$$

Rough estimates give that (47) is satisfied when

$$
e^{2 J}>4 e 3^{3 / 2}, \quad|\delta|<\frac{1}{12 e^{4 J}}
$$

this proves part $i$ ) of Lemma 2.1 in the + b.c. case.

The proof for periodic boundary conditions is nearly identical. The only difference is that now the maps $\sigma \mapsto \Gamma$ is two-to-one (i.e. $\sigma$ and $-\sigma$ yield the same $\Gamma$ ). Each $\Gamma$ is, as before, the union of p-connected contours $\gamma$ where the connection is the same as in the + b.c. case, and denote by $\mathcal{P}_{\Lambda}^{\text {per }}$ the set of all contour, which now also includes contours winding around the torus $\Lambda^{\text {per }}$. Therefore, we can write

$$
\pi_{G}^{p e r}\left(\left(f^{p e r}\right)^{k}\right)=\frac{2}{Z_{G}^{p e r}} e^{J\left|B_{\Lambda}\right|}\left(1+\delta e^{-4 J}\right)^{k|\Lambda|} \Xi_{\Lambda}^{p e r(k)}(J, \delta)
$$

where the factor 2 is due to the fact that a contour can be filled in two ways and

$$
\Xi_{\Lambda}^{p e r(k)}(J, \delta)=\sum_{n \geq 0} \frac{1}{n !} \sum_{\substack{\left(\gamma_{1}, \ldots, \gamma_{n}\right) \in\left(\mathcal{P}_{\Lambda}^{p e r}\right)^{n} \\ \gamma_{i} \sim \gamma_{j}}} \prod_{i=1}^{n}\left[e^{-2 J\left|\gamma_{i}\right|} \xi_{k}\left(\gamma_{i}\right)\right]
$$


This implies that

$$
\frac{1}{|\Lambda|} \ln \pi_{G}^{p e r}\left(\left(f^{p e r}\right)^{k}\right)=-\frac{\ln \left(Z_{G}^{p e r} / 2\right)}{|\Lambda|}+J \frac{\left|B_{\Lambda}\right|}{|\Lambda|}+k \ln \left(1+\delta e^{-4 J}\right)+\frac{1}{|\Lambda|} \ln \Xi_{\Lambda}^{p e r(k)}(J, \delta)
$$

with $\frac{1}{|\Lambda|} \ln \Xi_{\Lambda}^{\text {per(k)}}(J, \delta)$ being absolutely convergent if the same condition (44) is satisfied. Therefore $\frac{1}{|\Lambda|} \ln \pi_{G}^{\text {per }}\left(\left(f^{\text {per }}\right)^{k}\right)$ is analytic in $\delta$ uniformly in $\Lambda$ whenever $J$ and $\delta$ satisfy, as before, the condition given in (48).

Part ii)

The first order in $\delta$ of $\pi_{G}^{*}\left(\left(f^{*}\right)^{k}\right), k=1,2$, can be directly computed. Recalling (33), we have

$$
\begin{aligned}
\pi_{G}^{*}\left(\left(f^{*}\right)^{k}\right) & =\frac{\sum_{\sigma \in \mathcal{X}} w_{G}^{*}(\sigma)\left(\prod_{i \in \Lambda}\left(1+\delta \phi_{i}^{*}\right)\right)^{k}}{\sum_{\sigma \in \mathcal{X}} w_{G}^{*}(\sigma)}=\frac{\sum_{\sigma \in \mathcal{X}} w_{G}^{*}(\sigma)\left(1+k \sum_{i \in \Lambda} \delta \phi_{i}^{*}+O\left(\delta^{2}\right)\right)}{\sum_{\sigma \in \mathcal{X}} w_{G}^{*}(\sigma)}= \\
& =1+k \delta \frac{\sum_{\sigma \in \mathcal{X}} w_{G}^{*}(\sigma) \sum_{i \in \Lambda} \phi_{i}^{*}}{\sum_{\sigma \in \mathcal{X}} w_{G}^{*}(\sigma)}+O\left(\delta^{2}\right)=1+k \delta \sum_{i \in \Lambda} \pi_{G}^{*}\left(\phi_{i}^{*}\right)+O\left(\delta^{2}\right)
\end{aligned}
$$

Therefore, for $k=1,2$, we get

$$
\frac{1}{|\Lambda|} \pi_{G}^{*}\left(\left(f^{*}\right)^{k}\right)=k \delta \frac{\sum_{i \in \Lambda} \pi_{G}^{*}\left(\phi_{i}^{*}\right)}{|\Lambda|}+O\left(\delta^{2}\right)
$$

from which part $i i$ ) easily follows.

The proof of theorem 1.2 is similar, and even easier. Here we list the few changes needed in this case. We define

$$
\begin{gathered}
h_{i}^{I}(\sigma)=J\left(\sigma_{i \downarrow}+\sigma_{i^{\leftarrow}}\right), \\
\phi_{i}^{I}=e^{-2\left(h_{i}^{I}(\sigma) \sigma_{i}\right.}
\end{gathered}
$$

and

$$
f^{I}(\sigma)=\prod_{i \in \Lambda}\left(1+\delta \phi_{i}^{I}\right)
$$

The theorem follows exactly in the same way if we prove

Lemma 2.2 There exists $J_{c}$ such that for any $J>J_{c}$

i) $\quad \frac{\ln \pi_{G}^{p e r}\left(\left(f^{I}\right)^{2}\right)}{|\Lambda|}$ and $\frac{\ln \pi_{G}^{p e r}\left(f^{I}\right)}{|\Lambda|}$ are analytical functions of $\delta$ for $|\delta|<\delta_{J}$. 
ii) $\quad \frac{\ln \pi_{G}^{p e r}\left(\left(f^{I}\right)^{2}\right)}{|\Lambda|}-2 \frac{\ln \pi_{G}^{p e r}\left(f^{I}\right)}{|\Lambda|}=O\left(\delta^{2}\right)$

The proof of the lemma uses the same ideas. We have

$$
\pi_{G}^{p e r}\left(\left(f^{I}\right)^{k}\right)=\frac{1}{Z_{G}^{p e r}} e^{J\left|B_{\Lambda}\right|}\left(1+\delta e^{-4 J}\right)^{k|\Lambda|} \sum_{\Gamma \in \mathcal{G}_{\Lambda}}\left[e^{-2 J|\Gamma|} \xi_{k}^{I}(\Gamma)\right]
$$

where now, due to (50), (51)

$$
\xi_{k}^{I}(\Gamma)=\left[\frac{1+\delta}{1+\delta e^{-4 J}}\right]^{k\left|l_{1}(\Gamma)\right|}\left[\frac{1+\delta e^{+4 J}}{1+\delta e^{-4 J}}\right]^{k\left|l_{2}(\Gamma)\right|}
$$

Then we split $\Gamma$ in its connected components $\gamma_{1}, \ldots, \gamma_{n}$, where the notion of connection is in this case the usual one (since if $i \in l_{2}(\Gamma)$ then, due to (50), the two segments of $\Gamma$ at distance $1 / 2$ from $i$ must form an elbow.). Denoting by $\mathcal{P}_{\Lambda}^{I}$ (by $\mathcal{P}^{I}$ ) the set of standard contours in $\Lambda$ (in $\mathbb{Z}^{d}$ ), we can therefore write

$$
\sum_{\Gamma \in \mathcal{G}_{\Lambda}} e^{-2 J|\Gamma|} \xi_{k}^{I}(\Gamma)=\Xi_{\Lambda}^{I(k)}(J, \delta)=\sum_{n \geq 0} \frac{1}{n !} \sum_{\substack{\left(\gamma_{1}, \ldots, \gamma_{n}\right) \in\left(\mathcal{P}_{\Lambda}^{I}\right)^{n} \\ \gamma_{i} \sim \gamma_{j}}} \prod_{i=1}^{n}\left[e^{-2 J\left|\gamma_{i}\right|} \xi_{k}^{I}\left(\gamma_{i}\right)\right]
$$

So, as before, we have to analyze the absolute convergence of the logarithm of the quantity $\Xi_{\Lambda}^{I(k)}(J, \delta)$, the grand-canonical partition function of a a hard core polymer gas in which polymers are now standard contours in the set $\mathcal{P}_{\Lambda}$ with activity

$$
\rho_{k}^{I}(\gamma)=\xi_{k}^{I}(\gamma) e^{-2 J|\gamma|}
$$

and we have to prove that

$$
\sum_{\substack{\gamma \in \mathcal{P}^{\prime} \\ x \in \gamma}}\left|\rho_{k}^{I}(\gamma)\right| e^{|\gamma|} \leq 1
$$

where $\mathcal{P}$ is now the set of usual contours in $\mathbb{Z}^{2}$ and $x$ is any fixed vertex of $\mathbb{Z}^{2}$, due to the translation invariance of the model. We can easily find a bound for $\left|\rho_{k}^{I}(\gamma)\right|$ : we get, for $|\delta|<e^{4 J}$

$$
\rho_{k}^{I}(\gamma) \leq e^{-2 J|\gamma|}\left[\frac{1+|\delta| e^{+4 J}}{1-|\delta| e^{-4 J}}\right]^{k \sum_{s=1}^{2}\left|l_{s}(\gamma)\right|}
$$

Observing that in this case

$$
\sum_{s=1}^{2}\left|l_{s}(\gamma)\right| \leq|\gamma|
$$


we get, for any $k=1,2$

$$
\rho_{k}^{I}(\gamma) \leq e^{-2 J|\gamma|}\left[\frac{1+|\delta| e^{+4 J}}{1-|\delta| e^{-4 J}}\right]^{k|\gamma|} \leq e^{-2 J|\gamma|}\left[\frac{1+|\delta| e^{+4 J}}{1-|\delta| e^{-4 J}}\right]^{2|\gamma|}
$$

We call then

$$
A(J, \delta)=e^{-2 J}\left[\frac{1+|\delta| e^{+4 J}}{1-|\delta| e^{-4 J}}\right]^{2}
$$

and we obtain

$$
\sum_{\substack{\gamma \in \mathcal{P}^{\prime} \\ x \in \gamma}} \rho_{k}^{I}(\gamma) e^{|\gamma|} \leq \sum_{n \geq 4} A^{I}(J, \delta)^{n} e^{n} \sum_{\substack{\gamma \ni x \\|\gamma|=n}} 1 \leq \sum_{n \geq 4}\left[3 e A^{I}(J, \delta)\right]^{n}
$$

Here the factor $3^{n}$ comes form the usual estimate on Peierls contour. The rest of the proof is identical.

Acknowledgments: It is a pleasure to thank Giosi Benfatto for useful discussions. A.P. has been partially supported by the Brazilian agencies Conselho Nacional de Desenvolvimento Científico e Tecnológico $(\mathrm{CNPq})$ and Fundação de Amparo à Pesquisa do Estado de Minas Gerais (FAPEMIG - Programa de Pesquisador Mineiro). B.S. and E.S. thank the support of the A*MIDEX project (n. ANR-11-IDEX-0001-02) funded by the "Investissements d'Avenir" French Government program, managed by the French National Research Agency (ANR). B.S. has been supported by PRIN 2012, Problemi matematici in teoria cinetica ed applicazioni.

\section{References}

[1] R. Bissacot, R. Fernández, A. Procacci, On the convergence of cluster expansions for polymer gases, J. Statist. Phys. 139 (4), 598-617 (2010).

[2] E.N.M.Cirillo, F.R.NARd Metastability for Stochastic Dynamics with a Parallel Heat Bath Updating Rule, J. Statist. Phys. 110, 183-217 (2003).

[3] E.N.M.Cirillo, F.R.NARd, C.Spitoni Metastability ffor reversible Probabilistic Cellular Automata with self-interacton, J. Statist. Phys. 132, 431-471 (2008). 
[4] P.Dai Pra, B.Scoppola, E.Scoppola Sampling from a Gibbs measure with pair interaction by means of PCA J. Statist. Phys. 149, 722-737 (2012).

[5] P.Dai Pra, B.Scoppola, E.Scoppola Fast mixing for the low temperature 2D Ising model through irreversible parallel dynamics J. Statist. Phys. 159, 1-20 (2015).

[6] D. Dawson, A.,Synchronous and asynchronous reversible Markov systems, Canad. Math. Bull. 17 no. 5, 633-649 (1974/75).

[7] R.Fernandez, A. ProcacCi Cluster expansion for abstract polymer models. New bounds from an old approach Commun. Math. Phys. 274, 123-140 (2007).

[8] R. Fernandez, A. Toom, Non Gibbsiannes of the invariant measures of nonreversible cellular automata with totally asymmetric noise, Astérisque 287, 71-87 (2003).

[9] A.Gaudillière, B.Scoppola, E.Scoppola, M.Viale, Phase transition for the cavity approach to the clique problem on random graphs., J. Statist. Phys. 145, n. 5, 1127-1155 (2011).

[10] S. Goldstein , R. Kuik , J.L. Lebowitz, C. Maes, From PCAs to equilibrium systems and back., Commun. Math. Phys. 125, no. 1, 71-79 (1989).

[11] J. Z. Imbrie1, C. M. Newman, An Intermediate Phase with Slow Decay of Correlations in One Dimensional $1 /|i-j|^{2}$ Percolation, Ising and Potts Models, Commun. Math. Phys. 118, 303-336 (1988).

[12] A.Iovanella, B.Scoppola, E.Scoppola Some Spin Glass Ideas Applied to the Clique Problem, J. Statist. Phys. 126, n. 4, 895-915 (2007).

[13] R. Kotecký, D.Preiss, Cluster expansion for abstract polymer models, Commun. Math. Phys. 103, 491-498 (1986).

[14] O. Kozlov, N. Vasilyev, Reversible Markov chains with local interaction, Multicomponent random systems, Adv. Probab. Related Topics 6, Dekker, New York, pp. 451-469 (1980).

[15] H. KüNsch, Decay of Correlations under Dobrushin's Uniqueness Condition and its Applications, Commun. Math. Phys. 84, 207-222 (1982). 
[16] C. Lancia, B. Scoppola, Equilibrium and Non-equilibrium Ising Models by Means of PCA J. Statist. Phys. 153, 641-653 (2013).

[17] J.L. Lebowitz, C. Maes, E.R. Speer, Statistical mechanics of probabilistic cellular automata, J. Statist. Phys. 59, no. 1-2, 117-170 (1990).

[18] D.A.Levin, M.J.Luczak, Y.Peres Glauber dynamics for the mean-field Ising model: cut-off, critical power law and metastability, Probab. Theory Related Fields 146, 223-265 (2010).

[19] F.Martinelli Relaxation time of Markoc chains in statistical mechanics and combinatorial structures in "Probability on discrete structures", Encyclopedia of Mathematical Sciences, vol. 110 - Springer (2004).

[20] C.M. Newman, A general central limit theorem for FKG systems, Commun. Math. Phys. 91, n. 1, 75-80, (1983).

[21] E. Olivieri, M. E. VARes, Large deviations and metastability. Encyclopedia of Mathematics and its Applications, 100. Cambridge University Press, Cambridge, (2005).

[22] J.S.Wang, R.H.Swendsen Non universal critical dynamics in Monte Carlo simulations Phys. Rev. Lett. 58, 86-88 (1987). 